\title{
INEQUALITIES OF LIPSCHITZ TYPE FOR POWER SERIES IN BANACH ALGEBRAS
}

\author{
SEver S. Dragomir
}

Abstract. Let $f(z)=\sum_{n=0}^{\infty} \alpha_{n} z^{n}$ be a function defined by power series with complex coefficients and convergent on the open disk $D(0, R) \subset \mathbb{C}, R>0$. For any $x, y \in \mathcal{B}$, a Banach algebra, with $\|x\|,\|y\|<R$ we show among others that

$$
\|f(y)-f(x)\| \leq\|y-x\| \int_{0}^{1} f_{a}^{\prime}(\|(1-t) x+t y\|) d t
$$

where $f_{a}(z)=\sum_{n=0}^{\infty}\left|\alpha_{n}\right| z^{n}$. Inequalities for the commutator such as

$$
\|f(x) f(y)-f(y) f(x)\| \leq 2 f_{a}(M) f_{a}^{\prime}(M)\|y-x\|,
$$

if $\|x\|,\|y\| \leq M<R$, as well as some inequalities of Hermite-Hadamard type are also provided.

\section{Introduction}

Let $\mathcal{B}$ be an algebra. An algebra norm on $\mathcal{B}$ is a map $\|\cdot\|: \mathcal{B} \rightarrow[0, \infty)$ such that $(\mathcal{B},\|\cdot\|)$ is a normed space, and, further:

$$
\|a b\| \leq\|a\|\|b\|
$$

Received: 21.10.2014. Revised: 4.03.2015.

(2010) Mathematics Subject Classification: 47A63; 47A99.

Key words and phrases: Banach algebras, Power series, Lipschitz type inequalities, Hermite-Hadamard type inequalities. 
for any $a, b \in \mathcal{B}$. The normed algebra $(\mathcal{B},\|\cdot\|)$ is a Banach algebra if $\|\cdot\|$ is a complete norm.

We assume that the Banach algebra is unital, this means that $\mathcal{B}$ has an identity 1 and that $\|1\|=1$.

Let $\mathcal{B}$ be a unital algebra. An element $a \in \mathcal{B}$ is invertible if there exists an element $b \in \mathcal{B}$ with $a b=b a=1$. The element $b$ is unique; it is called the inverse of $a$ and written $a^{-1}$ or $\frac{1}{a}$. The set of invertible elements of $\mathcal{B}$ is denoted by $\operatorname{Inv} \mathcal{B}$. If $a, b \in \operatorname{Inv} \mathcal{B}$ then $a b \in \operatorname{Inv} \mathcal{B}$ and $(a b)^{-1}=b^{-1} a^{-1}$.

For a unital Banach algebra we also have:

(i) If $a \in \mathcal{B}$ and $\lim _{n \rightarrow \infty}\left\|a^{n}\right\|^{1 / n}<1$, then $1-a \in \operatorname{Inv} \mathcal{B}$;

(ii) $\{a \in \mathcal{B}:\|1-b\|<1\} \subset \operatorname{Inv} \mathcal{B}$;

(iii) Inv $\mathcal{B}$ is an open subset of $\mathcal{B}$;

(iv) The map $\operatorname{Inv} \mathcal{B} \ni a \longmapsto a^{-1} \in \operatorname{Inv} \mathcal{B}$ is continuous.

For simplicity, we denote $z 1$, where $z \in \mathbb{C}$ and 1 is the identity of $\mathcal{B}$, by $z$. The resolvent set of $a \in \mathcal{B}$ is defined by

$$
\rho(a):=\{z \in \mathbb{C}: z-a \in \operatorname{Inv} \mathcal{B}\}
$$

the spectrum of $a$ is $\sigma(a)$, the complement of $\rho(a)$ in $\mathbb{C}$, and the resolvent function of $a$ is $R_{a}: \rho(a) \rightarrow \operatorname{Inv} \mathcal{B}$,

$$
R_{a}(z):=(z-a)^{-1} .
$$

For each $z, w \in \rho(a)$ we have the identity

$$
R_{a}(w)-R_{a}(z)=(z-w) R_{a}(z) R_{a}(w) .
$$

We also have that

$$
\sigma(a) \subset\{z \in \mathbb{C}: \quad|z| \leq\|a\|\} .
$$

The spectral radius of $a$ is defined as

$$
\nu(a)=\sup \{|z|: z \in \sigma(a)\} .
$$

If $a, b$ are commuting elements in $\mathcal{B}$, i.e. $a b=b a$, then

$$
\nu(a b) \leq \nu(a) \nu(b) \text { and } \nu(a+b) \leq \nu(a)+\nu(b) .
$$

Let $\mathcal{B}$ be a unital Banach algebra and $a \in \mathcal{B}$. Then

(i) The resolvent set $\rho(a)$ is open in $\mathbb{C}$;

(ii) For any bounded linear functionals $\lambda: \mathcal{B} \rightarrow \mathbb{C}$, the function $\lambda \circ R_{a}$ is analytic on $\rho(a)$; 
(iii) The spectrum $\sigma(a)$ is compact and nonempty in $\mathbb{C}$;

(iv) For each $n \in \mathbb{N}$ and $r>\nu(a)$, we have

$$
a^{n}=\frac{1}{2 \pi i} \int_{|\xi|=r} \xi^{n}(\xi-a)^{-1} d \xi
$$

(v) We have

$$
\nu(a)=\lim _{n \rightarrow \infty}\left\|a^{n}\right\|^{1 / n} .
$$

Let $f$ be an analytic functions on the open disk $D(0, R)$ given by the power series

$$
f(z):=\sum_{j=0}^{\infty} \alpha_{j} z^{j}, \quad|z|<R .
$$

If $\nu(a)<R$, then the series $\sum_{j=0}^{\infty} \alpha_{j} a^{j}$ converges in the Banach algebra $\mathcal{B}$ because $\sum_{j=0}^{\infty}\left|\alpha_{j}\right|\left\|a^{j}\right\|<\infty$, and we can define $f(a)$ to be its sum. Clearly $f(a)$ is well defined and there are many examples of important functions on a Banach algebra $\mathcal{B}$ that can be constructed in this way. For instance, the exponential map on $\mathcal{B}$ denoted exp and defined as

$$
\exp a:=\sum_{j=0}^{\infty} \frac{1}{j !} a^{j} \quad \text { for each } a \in \mathcal{B} .
$$

If $\mathcal{B}$ is not commutative, then many of the familiar properties of the exponential function from the scalar case do not hold. The following key formula is valid, however with the additional hypothesis of commutativity for $a$ and $b$ from $\mathcal{B}$

$$
\exp (a+b)=\exp (a) \exp (b) .
$$

In a general Banach algebra $\mathcal{B}$ it is difficult to determine the elements in the range of the exponential map $\exp (\mathcal{B})$, i.e. the element which have a "logarithm". However, it is easy to see that if $a$ is an element in $B$ such that $\|1-a\|<1$, then $a$ is in $\exp (\mathcal{B})$. That follows from the fact that if we set

$$
b=-\sum_{n=1}^{\infty} \frac{1}{n}(1-a)^{n},
$$

then the series converges absolutely and, as in the scalar case, substituting this series into the series expansion for $\exp (b)$ yields $\exp (b)=a$. 
In this paper we establish some upper bounds for the following quantities

$$
\begin{gathered}
\|f(y)-f(x)\|, \\
\|f(x) f(y)-f(y) f(x)\|, \\
\left\|f\left(\frac{x+y}{2}\right)-\int_{0}^{1} f((1-s) x+s y) d s\right\|
\end{gathered}
$$

and

$$
\left\|\frac{f(x)+f(y)}{2}-\int_{0}^{1} f((1-s) x+s y) d s\right\|
$$

that can naturally be associated with the analytic functions $f(z):=\sum_{j=0}^{\infty} \alpha_{j} z^{j}$ defined on the open disk $D(0, R)$ and the elements $x$ and $y$ of the unital Banach algebra $\mathcal{B}$. Some applications for functions of interest such as the exponential map on $\mathcal{B}$ are provided as well.

\section{Lipschitz type inequalities}

Now, by the help of power series $f(z)=\sum_{n=0}^{\infty} \alpha_{n} z^{n}$ we can naturally construct another power series which will have as coefficients the absolute values of the coefficients of the original series, namely, $f_{a}(z):=\sum_{n=0}^{\infty}\left|\alpha_{n}\right| z^{n}$. It is obvious that this new power series will have the same radius of convergence as the original series. We also notice that if all coefficients $\alpha_{n} \geq 0$, then $f_{a}=f$.

The following result is valid.

Theorem 1. Let $f(z)=\sum_{n=0}^{\infty} \alpha_{n} z^{n}$ be a function defined by power series with complex coefficients and convergent on the open disk $D(0, R) \subset \mathbb{C}, R>0$. For any $x, y \in \mathcal{B}$ with $\|x\|,\|y\|<R$ we have

$$
\|f(y)-f(x)\| \leq\|y-x\| \int_{0}^{1} f_{a}^{\prime}(\|(1-t) x+t y\|) d t .
$$


Proof. We use the identity (see for instance [2, p. 254])

$$
a^{n}-b^{n}=\sum_{j=0}^{n-1} a^{n-1-j}(a-b) b^{j}
$$

that holds for any $a, b \in \mathcal{B}$ and $n \geq 1$.

For $x, y \in \mathcal{B}$ we consider the function $\varphi:[0,1] \rightarrow \mathcal{B}$ defined by $\varphi(t)=$ $[(1-t) x+t y]^{n}$. For $t \in(0,1)$ and $\varepsilon \neq 0$ with $t+\varepsilon \in(0,1)$ we have from 2.2 that

$$
\begin{aligned}
\varphi(t+\varepsilon)-\varphi(t) & =[(1-t-\varepsilon) x+(t+\varepsilon) y]^{n}-[(1-t) x+t y]^{n} \\
& =\varepsilon \sum_{j=0}^{n-1}[(1-t-\varepsilon) x+(t+\varepsilon) y]^{n-1-j}(y-x)[(1-t) x+t y]^{j} .
\end{aligned}
$$

Dividing with $\varepsilon \neq 0$ and taking the limit over $\varepsilon \rightarrow 0$ we have in the norm topology of $\mathcal{B}$ that

$$
\begin{aligned}
\varphi^{\prime}(t) & =\lim _{\varepsilon \rightarrow 0} \frac{1}{\varepsilon}[\varphi(t+\varepsilon)-\varphi(t)] \\
& =\sum_{j=0}^{n-1}[(1-t) x+t y]^{n-1-j}(y-x)[(1-t) x+t y]^{j} .
\end{aligned}
$$

Integrating on $[0,1]$ we get from $(2.3)$ that

$$
\int_{0}^{1} \varphi^{\prime}(t) d t=\sum_{j=0}^{n-1} \int_{0}^{1}[(1-t) x+t y]^{n-1-j}(y-x)[(1-t) x+t y]^{j} d t
$$

and since

$$
\int_{0}^{1} \varphi^{\prime}(t) d t=\varphi(1)-\varphi(0)=y^{n}-x^{n}
$$

then we get the following equality of interest

$$
y^{n}-x^{n}=\sum_{j=0}^{n-1} \int_{0}^{1}[(1-t) x+t y]^{n-1-j}(y-x)[(1-t) x+t y]^{j} d t
$$

for any $x, y \in \mathcal{B}$ and $n \geq 1$. 
Taking the norm and utilizing the properties of Bochner integral for vector valued functions (see for instance [18, p. 21]) we have

$$
\begin{aligned}
\left\|y^{n}-x^{n}\right\| & \leq \sum_{j=0}^{n-1}\left\|\int_{0}^{1}[(1-t) x+t y]^{n-1-j}(y-x)[(1-t) x+t y]^{j} d t\right\| \\
& \leq \sum_{j=0}^{n-1} \int_{0}^{1}\left\|[(1-t) x+t y]^{n-1-j}(y-x)[(1-t) x+t y]^{j}\right\| d t \\
& \leq \sum_{j=0}^{n-1} \int_{0}^{1}\|(1-t) x+t y\|^{n-1-j}\|y-x\|\|(1-t) x+t y\|^{j} d t \\
& =n\|y-x\| \int_{0}^{1}\|(1-t) x+t y\|^{n-1} d t
\end{aligned}
$$

for any $x, y \in \mathcal{B}$ and $n \geq 1$.

Now, for any $m \geq 1$, by making use of the inequality $(2.2)$ we have

$$
\begin{aligned}
\left\|\sum_{n=0}^{m} \alpha_{n} y^{n}-\sum_{n=0}^{m} \alpha_{n} x^{n}\right\| & =\left\|\sum_{n=1}^{m} \alpha_{n}\left(y^{n}-x^{n}\right)\right\| \\
& \leq \sum_{n=1}^{m}\left|\alpha_{n}\right|\left\|y^{n}-x^{n}\right\| \\
& \leq\|y-x\| \sum_{n=1}^{m} n\left|\alpha_{n}\right| \int_{0}^{1}\|(1-t) x+t y\|^{n-1} d t \\
& =\|y-x\| \int_{0}^{1} \sum_{n=1}^{m} n\left|\alpha_{n}\right|\|(1-t) x+t y\|^{n-1} d t .
\end{aligned}
$$

Moreover, since $\|x\|,\|y\|<R$, then the series $\sum_{n=0}^{\infty} \alpha_{n} y^{n}, \sum_{n=0}^{\infty} \alpha_{n} x^{n}$ and

$$
\sum_{n=1}^{\infty} n\left|\alpha_{n}\right|\|(1-t) x+t y\|^{n-1}
$$

are convergent and

$$
\sum_{n=0}^{\infty} \alpha_{n} y^{n}=f(y), \quad \sum_{n=0}^{\infty} \alpha_{n} x^{n}=f(x)
$$


while

$$
\sum_{n=1}^{\infty} n\left|\alpha_{n}\right|\|(1-t) x+t y\|^{n-1}=f_{a}^{\prime}(\|(1-t) x+t y\|)
$$

Therefore, by taking the limit over $m \rightarrow \infty$ in the inequality 2.5 we deduce the desired result (2.1).

REMARK 1. We observe that $f_{a}^{\prime}$ is monotonic nondecreasing and convex on the interval $[0, R)$ and since the function $\psi(t):=\|(1-t) x+t y\|$ is convex on $[0,1]$ we have that $f_{a}^{\prime} \circ \psi$ is also convex on $[0,1]$. Utilising the HermiteHadamard inequality for convex functions (see for instance [13, p. 2]) we have the sequence of inequalities

$$
\begin{aligned}
\|f(y)-f(x)\| & \leq\|y-x\| \int_{0}^{1} f_{a}^{\prime}(\|(1-t) x+t y\|) d t \\
& \leq \frac{1}{2}\|y-x\|\left[f_{a}^{\prime}\left(\left\|\frac{x+y}{2}\right\|\right)+\frac{f_{a}^{\prime}(\|x\|)+f_{a}^{\prime}(\|y\|)}{2}\right] \\
& \leq \frac{1}{2}\|y-x\|\left[f_{a}^{\prime}(\|x\|)+f_{a}^{\prime}(\|y\|)\right] \\
& \leq\|y-x\| \max \left\{f_{a}^{\prime}(\|x\|), f_{a}^{\prime}(\|y\|)\right\} .
\end{aligned}
$$

We also have

$$
\begin{aligned}
\|f(y)-f(x)\| & \leq\|y-x\| \int_{0}^{1} f_{a}^{\prime}(\|(1-t) x+t y\|) d t \\
& \leq\|y-x\| \int_{0}^{1} f_{a}^{\prime}((1-t)\|x\|+t\|y\|) d t \\
& \leq \frac{1}{2}\|y-x\|\left[f_{a}^{\prime}\left(\frac{\|x\|+\|y\|}{2}\right)+\frac{f_{a}^{\prime}(\|x\|)+f_{a}^{\prime}(\|y\|)}{2}\right] \\
& \leq \frac{1}{2}\|y-x\|\left[f_{a}^{\prime}(\|x\|)+f_{a}^{\prime}(\|y\|)\right] \\
& \leq\|y-x\| \max \left\{f_{a}^{\prime}(\|x\|), f_{a}^{\prime}(\|y\|)\right\} .
\end{aligned}
$$


We observe that if $\|y\| \neq\|x\|$, then by the change of variable $s=(1-t)\|x\|+$ $t\|y\|$ we have

$$
\begin{aligned}
\int_{0}^{1} f_{a}^{\prime}((1-t)\|x\|+t\|y\|) d t & =\frac{1}{\|y\|-\|x\|} \int_{\|x\|}^{\|y\|} f_{a}^{\prime}(s) d s \\
& =\frac{f_{a}(\|y\|)-f_{a}(\|x\|)}{\|y\|-\|x\|} .
\end{aligned}
$$

If $\|y\|=\|x\|$, then

$$
\int_{0}^{1} f_{a}^{\prime}((1-t)\|x\|+t\|y\|) d t=f_{a}^{\prime}(\|x\|) .
$$

Utilising these observations we then get the following divided difference inequality for $x \neq y$

$$
\begin{aligned}
\frac{\|f(y)-f(x)\|}{\|y-x\|} & \leq \int_{0}^{1} f_{a}^{\prime}(\|(1-t) x+t y\|) d t \\
& \leq \begin{cases}\frac{f_{a}(\|y\|)-f_{a}(\|x\|)}{\|y\|-\|x\|} & \text { if }\|y\| \neq\|x\|, \\
f_{a}^{\prime}(\|x\|) & \text { if }\|y\|=\|x\| .\end{cases}
\end{aligned}
$$

If $\|x\|,\|y\| \leq M<R$, then from either of the inequalities (2.6) or (2.7) we have the Lipschitz type inequality

$$
\|f(y)-f(x)\| \leq f_{a}^{\prime}(M)\|y-x\| .
$$

REMARK 2. We observe that the integral $\int_{0}^{1} f_{a}^{\prime}(\|(1-t) x+t y\|) d t$, which might be difficult to compute in various examples of Banach algebras, has got the simpler bounds

$$
B_{1}(x, y):=\frac{1}{2}\left[f_{a}^{\prime}\left(\left\|\frac{x+y}{2}\right\|\right)+\frac{f_{a}^{\prime}(\|x\|)+f_{a}^{\prime}(\|y\|)}{2}\right]
$$

and

$$
B_{2}(x, y):= \begin{cases}\frac{f_{a}(\|y\|)-f_{a}(\|x\|)}{\|y\|-\|x\|} & \text { if }\|y\| \neq\|x\|, \\ f_{a}^{\prime}(\|x\|) & \text { if }\|y\|=\|x\| .\end{cases}
$$

It is natural then to ask which of these bounds is better? 
Let us consider the simple examples of powers, namely $f(z)=z^{m}$ with $m \geq 1$. Then

$$
B_{1}(x, y)=\frac{1}{2} m\left[\left\|\frac{x+y}{2}\right\|^{m-1}+\frac{\|x\|^{m-1}+\|y\|^{m-1}}{2}\right]
$$

and

$$
B_{2}(x, y):= \begin{cases}\|y\|^{m-1}+\|y\|^{m-2}\|x\|+\ldots+\|x\|^{m-1} & \text { if }\|y\| \neq\|x\|, \\ m\|x\|^{m-1} & \text { if }\|y\|=\|x\| .\end{cases}
$$

If we take $y=t x$ with $\|x\|=1$ and $|t| \neq 1$ then we get

$$
B_{1}(t)=\frac{1}{2} m\left[\left|\frac{1+t}{2}\right|^{m-1}+\frac{1+|t|^{m-1}}{2}\right]
$$

and

$$
B_{2}(t)=|t|^{m-1}+\ldots+|t|+1 .
$$

If we take $m=4$ and plot the difference

$$
d(t):=2\left(\left|\frac{t+1}{2}\right|^{3}+\frac{1+|t|^{3}}{2}\right)-\left(|t|^{3}+|t|^{2}+|t|+1\right)
$$

on the interval $[-8,8]$, then we can conclude that some time the first bound is better than the second, while other time the conclusion is the other way around.

The plot for the function $d$ is depicted in the Figure 2 below.

It is natural now to consider some examples of interest.

If we consider the exponential function $\exp (z)=\sum_{n=0}^{\infty} \frac{1}{n !} z^{n}$, then for any $x, y \in \mathcal{B}$ we have the inequalities

$$
\begin{aligned}
\|\exp (y)-\exp (x)\| & \leq\|y-x\| \int_{0}^{1} \exp (\|(1-t) x+t y\|) d t \\
& \leq\|y-x\|\left\{\begin{array}{l}
\frac{1}{2}\left[\exp \left(\left\|\frac{x+y}{2}\right\|\right)+\frac{\exp (\|x\|)+\exp (\|y\|)}{2}\right], \\
\frac{\exp (\|y\|)-\exp (\|x\|)}{\|y\|-\|x\|} \text { if }\|y\| \neq\|x\|, \\
\exp (\|x\|) \text { if }\|y\|=\|x\| .
\end{array}\right.
\end{aligned}
$$




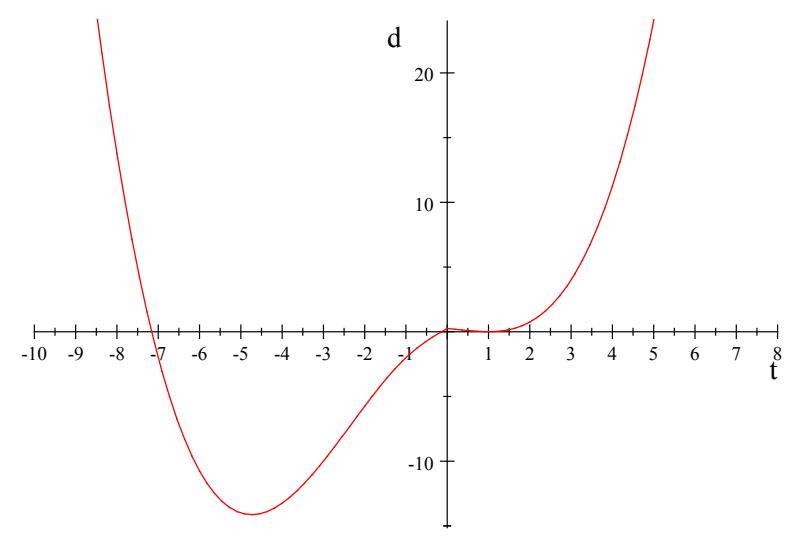

Figure 1. Variation of the difference $d(t)$ for $t \in[-8,8]$

Now, if we consider the functions $(1-z)^{-1}=\sum_{n=0}^{\infty} z^{n}$ and $(1+z)^{-1}=$ $\sum_{n=0}^{\infty}(-1)^{n} z^{n}$, then for any $x, y \in \mathcal{B}$ with $\|x\|,\|y\|<1$ we have the inequalities

$$
\begin{aligned}
\left\|(1 \pm y)^{-1}-(1 \pm x)^{-1}\right\| \leq\|y-x\| \int_{0}^{1}(1-\|(1-t) x+t y\|)^{-2} d t \\
\leq\|y-x\|\left\{\begin{array}{l}
\frac{1}{2}\left[\left(1-\left\|\frac{x+y}{2}\right\|\right)^{-2}+\frac{(1-\|x\|)^{-2}+(1-\|y\|)^{-2}}{2}\right] \\
(1-\|y\|)^{-1}(1-\|x\|)^{-1} \text { if }\|y\| \neq\|x\|, \\
(1-\|x\|)^{-2} \text { if }\|y\|=\|x\| .
\end{array}\right.
\end{aligned}
$$

\section{Inequalities for Commutators}

By the use of Lipschitz type inequalities obtained before we can establish some upper bounds for the commutator

$$
f(x) g(y)-g(y) f(x)
$$

where $x, y \in \mathcal{B}$ with $\|x\|,\|y\|<R$.

ThEOREM 2. Let $f(z)=\sum_{n=0}^{\infty} \alpha_{n} z^{n}$ and $g(z)=\sum_{n=0}^{\infty} \beta_{n} z^{n}$ be two functions defined by power series with complex coefficients and convergent on the 
open disk $D(0, R) \subset \mathbb{C}, R>0$. For any $x, y \in \mathcal{B}$ with $\|x\|,\|y\|<R$ we have

$$
\begin{aligned}
& \|f(x) g(y)-g(y) f(x)\| \\
& \leq\|y-x\|\left[\min \left\{f_{a}(\|x\|), f_{a}(\|y\|)\right\} \int_{0}^{1} g_{a}^{\prime}(\|(1-t) x+t y\|) d t\right. \\
& \left.\quad+\min \left\{g_{a}(\|x\|), g_{a}(\|y\|)\right\} \int_{0}^{1} f_{a}^{\prime}(\|(1-t) x+t y\|) d t\right] \\
& \leq\|y-x\|\left[\frac{f_{a}(\|x\|)+f_{a}(\|y\|)}{2} \int_{0}^{1} g_{a}^{\prime}(\|(1-t) x+t y\|) d t\right. \\
& \left.\quad+\frac{g_{a}(\|x\|)+g_{a}(\|y\|)}{2} \int_{0}^{1} f_{a}^{\prime}(\|(1-t) x+t y\|) d t\right] .
\end{aligned}
$$

Proof. Let $n, m \in \mathbb{N}$. Then we have

$$
\begin{aligned}
x^{n} y^{m}-y^{n} x^{m} & =x^{n} y^{m}-x^{n} x^{m}+x^{n} x^{m}-y^{n} x^{m} \\
& =x^{n}\left(y^{m}-x^{m}\right)+\left(x^{n}-y^{n}\right) x^{m} .
\end{aligned}
$$

Utilising the properties of the norm, we have

$$
\begin{aligned}
\left\|x^{n} y^{m}-y^{n} x^{m}\right\| & \leq\left\|x^{n}\left(y^{m}-x^{m}\right)\right\|+\left\|\left(x^{n}-y^{n}\right) x^{m}\right\| \\
& \leq\left\|x^{n}\right\|\left\|y^{m}-x^{m}\right\|+\left\|x^{n}-y^{n}\right\|\left\|x^{m}\right\| \\
& \leq\|x\|^{n}\left\|y^{m}-x^{m}\right\|+\|x\|^{m}\left\|x^{n}-y^{n}\right\|
\end{aligned}
$$

for any $n, m \in \mathbb{N}$.

We also have

$$
x^{n} y^{m}-y^{n} x^{m}=y^{n}\left(y^{m}-x^{m}\right)+\left(x^{n}-y^{n}\right) y^{m}
$$

which gives

$$
\left\|x^{n} y^{m}-y^{n} x^{m}\right\| \leq\|y\|^{n}\left\|y^{m}-x^{m}\right\|+\|y\|^{m}\left\|x^{n}-y^{n}\right\|
$$

for any $n, m \in \mathbb{N}$.

Therefore

$$
\begin{aligned}
\left\|x^{n} y^{m}-y^{n} x^{m}\right\| & \leq \min \left\{\|x\|^{n},\|y\|^{n}\right\}\left\|y^{m}-x^{m}\right\| \\
& +\min \left\{\|x\|^{m},\|y\|^{m}\right\}\left\|y^{n}-x^{n}\right\|
\end{aligned}
$$

for any $n, m \in \mathbb{N}$. 
For any $k \geq 1$ we then have

$$
\begin{aligned}
\| & \sum_{n=0}^{k} \alpha_{n} x^{n} \sum_{m=0}^{k} \beta_{m} y^{m}-\sum_{n=0}^{k} \alpha_{n} y^{n} \sum_{m=0}^{k} \beta_{m} x^{m} \| \\
= & \left\|\sum_{n=0}^{k} \sum_{m=0}^{k} \alpha_{n} \beta_{m}\left(x^{n} y^{m}-y^{n} x^{m}\right)\right\| \\
\leq & \sum_{n=0}^{k} \sum_{m=0}^{k}\left|\alpha_{n}\right|\left|\beta_{m}\right|\left\|x^{n} y^{m}-y^{n} x^{m}\right\| \\
\leq & \sum_{n=0}^{k}\left|\alpha_{n}\right| \min \left\{\|x\|^{n},\|y\|^{n}\right\} \sum_{m=0}^{k}\left|\beta_{m}\right|\left\|y^{m}-x^{m}\right\| \\
& +\sum_{n=0}^{k}\left|\beta_{m}\right| \min \left\{\|x\|^{m},\|y\|^{m}\right\} \sum_{m=0}^{k}\left|\alpha_{n}\right|\left\|y^{n}-x^{n}\right\| \\
\leq & \min \left\{\sum_{n=0}^{k}\left|\alpha_{n}\right|\|x\|^{n}, \sum_{n=0}^{k}\left|\alpha_{n}\right|\|y\|^{n}\right\} \sum_{m=0}^{k}\left|\beta_{m}\right|\left\|y^{m}-x^{m}\right\| \\
& +\min \left\{\sum_{n=0}^{k}\left|\beta_{m}\right|\|x\|^{m}, \sum_{n=0}^{k}\left|\beta_{m}\right|\|y\|^{m}\right\} \sum_{m=0}^{k}\left|\alpha_{n}\right|\left\|y^{n}-x^{n}\right\|
\end{aligned}
$$

for any $x, y \in \mathcal{B}$ with $\|x\|,\|y\|<R$.

From 2.5 we have

$$
\begin{aligned}
\sum_{m=0}^{k}\left|\beta_{m}\right|\left\|y^{m}-x^{m}\right\| & =\sum_{m=1}^{k}\left|\beta_{m}\right|\left\|y^{m}-x^{m}\right\| \\
& \leq\|y-x\| \int_{0}^{1} \sum_{m=1}^{k} m\left|\beta_{m}\right|\|(1-t) x+t y\|^{m-1} d t
\end{aligned}
$$

and

$$
\sum_{m=0}^{k}\left|\alpha_{n}\right|\left\|y^{n}-x^{n}\right\| \leq\|y-x\| \int_{0}^{1} \sum_{n=1}^{k} n\left|\beta_{n}\right|\|(1-t) x+t y\|^{n-1} d t
$$

for any $x, y \in \mathcal{B}$ with $\|x\|,\|y\|<R$. 
From $3.2-3.4$ we have

$$
\left\|\sum_{n=0}^{k} \alpha_{n} x^{n} \sum_{m=0}^{k} \beta_{m} y^{m}-\sum_{n=0}^{k} \alpha_{n} y^{n} \sum_{m=0}^{k} \beta_{m} x^{m}\right\| \leq\|y-x\|
$$

$\times\left\{\min \left\{\sum_{n=0}^{k}\left|\alpha_{n}\right|\|x\|^{n}, \sum_{n=0}^{k}\left|\alpha_{n}\right|\|y\|^{n}\right\} \int_{0}^{1} \sum_{m=1}^{k} m\left|\beta_{m}\right| \|(1-t) x+\left.t y\right|^{m-1} d t\right.$

$\left.+\min \left\{\sum_{n=0}^{k}\left|\beta_{m}\right|\|x\|^{m}, \sum_{n=0}^{k}\left|\beta_{m}\right|\|y\|^{m}\right\} \int_{0}^{1} \sum_{n=1}^{k} n\left|\beta_{n}\right|\|(1-t) x+t y\|^{n-1} d t\right\}$

for any $x, y \in \mathcal{B}$ with $\|x\|,\|y\|<R$ and $k \geq 1$.

Since all the series whose partial sums are involved in 3.5 are convergent, then by letting $m \rightarrow \infty$ in 3.5 we deduce the first inequality in (3.1).

The second inequality is obvious.

REMARK 3. If $g=f$ in (3.1), then we have the following sequence of inequalities

$$
\begin{aligned}
& \|f(x) f(y)-f(y) f(x)\| \\
& \leq 2\|y-x\| \min \left\{f_{a}(\|x\|), f_{a}(\|y\|)\right\} \int_{0}^{1} f_{a}^{\prime}(\|(1-t) x+t y\|) d t \\
& \leq\|y-x\| \min \left\{f_{a}(\|x\|), f_{a}(\|y\|)\right\} \\
& \times\left[f_{a}^{\prime}\left(\left\|\frac{x+y}{2}\right\|\right)+\frac{f_{a}^{\prime}(\|x\|)+f_{a}^{\prime}(\|y\|)}{2}\right] \\
& \leq\|y-x\| \min \left\{f_{a}(\|x\|), f_{a}(\|y\|)\right\}\left[f_{a}^{\prime}(\|x\|)+f_{a}^{\prime}(\|y\|)\right] \\
& \leq 2\|y-x\| \min \left\{f_{a}(\|x\|), f_{a}(\|y\|)\right\} \max \left\{f_{a}^{\prime}(\|x\|), f_{a}^{\prime}(\|y\|)\right\}
\end{aligned}
$$

and

$$
\begin{aligned}
& \|f(x) f(y)-f(y) f(x)\| \\
& \leq 2\|y-x\| \min \left\{f_{a}(\|x\|), f_{a}(\|y\|)\right\} \int_{0}^{1} f_{a}^{\prime}(\|(1-t) x+t y\|) d t \\
& \leq 2\|y-x\| \min \left\{f_{a}(\|x\|), f_{a}(\|y\|)\right\} \int_{0}^{1} f_{a}^{\prime}((1-t)\|x\|+t\|y\|) d t
\end{aligned}
$$




$$
\begin{aligned}
\leq & \|y-x\| \min \left\{f_{a}(\|x\|), f_{a}(\|y\|)\right\} \\
& \times\left[f_{a}^{\prime}\left(\frac{\|x\|+\|y\|}{2}\right)+\frac{f_{a}^{\prime}(\|x\|)+f_{a}^{\prime}(\|y\|)}{2}\right] \\
\leq & \|y-x\| \min \left\{f_{a}(\|x\|), f_{a}(\|y\|)\right\}\left[f_{a}^{\prime}(\|x\|)+f_{a}^{\prime}(\|y\|)\right] \\
\leq & 2\|y-x\| \min \left\{f_{a}^{\prime}(\|x\|), f_{a}^{\prime}(\|y\|)\right\} \max \left\{f_{a}^{\prime}(\|x\|), f_{a}^{\prime}(\|y\|)\right\}
\end{aligned}
$$

for any $x, y \in \mathcal{B}$ with $\|x\|,\|y\|<R$.

Since

$$
\min \left\{f_{a}(\|x\|), f_{a}(\|y\|)\right\} \leq \frac{f_{a}(\|x\|)+f_{a}(\|y\|)}{2}
$$

and

$$
\int_{0}^{1} f_{a}^{\prime}((1-t)\|x\|+t\|y\|) d t= \begin{cases}\frac{f_{a}(\|y\|)-f_{a}(\|x\|)}{\|y\|-\|x\|} & \text { if }\|y\| \neq\|x\|, \\ f_{a}^{\prime}(\|x\|) & \text { if }\|y\|=\|x\|,\end{cases}
$$

then by the second inequality in 3.7 we have for $x \neq y$ that

$$
\begin{aligned}
& \frac{\|f(x) f(y)-f(y) f(x)\|}{\|y-x\|} \\
& \leq 2 \min \left\{f_{a}(\|x\|), f_{a}(\|y\|)\right\} \int_{0}^{1} f_{a}^{\prime}(\|(1-t) x+t y\|) d t \\
& \leq \begin{cases}\frac{f_{a}^{2}(\|y\|)-f_{a}^{2}(\|x\|)}{\|y\|-\|x\|} & \text { if }\|y\| \neq\|x\|, \\
2 f_{a}(\|x\|) f_{a}^{\prime}(\|x\|) & \text { if }\|y\|=\|x\| .\end{cases}
\end{aligned}
$$

If $\|x\|,\|y\| \leq M<R$, then we can state the simpler inequality

$$
\|f(x) f(y)-f(y) f(x)\| \leq 2 f_{a}(M) f_{a}^{\prime}(M)\|y-x\| .
$$

Now, if for instance we use the first part of the inequality (3.6) for the exponential function, then we get

$$
\begin{aligned}
& \|\exp (x) \exp (y)-\exp (y) \exp (x)\| \\
& \leq 2\|y-x\| \min \{\exp (\|x\|), \exp (\|y\|)\} \int_{0}^{1} \exp (\|(1-t) x+t y\|) d t \\
& \leq\|y-x\| \min \{\exp (\|x\|), \exp (\|y\|)\} \\
& \quad \times\left[\exp \left(\left\|\frac{x+y}{2}\right\|\right)+\frac{\exp (\|x\|)+\exp (\|y\|)}{2}\right]
\end{aligned}
$$


while from the first part of 3.8 we have

$$
\begin{aligned}
& \|\exp (x) \exp (y)-\exp (y) \exp (x)\| \\
& \leq 2\|y-x\| \min \{\exp (\|x\|), \exp (\|y\|)\} \int_{0}^{1} \exp (\|(1-t) x+t y\|) d t \\
& \leq\|y-x\| \begin{cases}\frac{\exp (2\|y\|)-\exp (2\|x\|)}{\|y\|-\|x\|} & \text { if }\|y\| \neq\|x\|, \\
2 \exp (2\|x\|) & \text { if }\|y\|=\|x\|,\end{cases}
\end{aligned}
$$

for any $x, y \in \mathcal{B}$.

\section{Applications for Hermite-Hadamard type inequalities}

The following result is well known in the Theory of Inequalities as the Hermite-Hadamard inequality

$$
f\left(\frac{a+b}{2}\right) \leq \frac{1}{b-a} \int_{a}^{b} f(t) d t \leq \frac{f(a)+f(b)}{2}
$$

for any convex function $f:[a, b] \rightarrow \mathbb{R}$. For numerous results related to this inequality, see the monograph [13].

The distance between the middle and the left term for Lipschitzian functions with the constant $L>0$ has been estimated in [4] to be

$$
\left|\frac{1}{b-a} \int_{a}^{b} f(t) d t-f\left(\frac{a+b}{2}\right)\right| \leq \frac{1}{4} L(b-a)
$$

while the distance between the right term and the middle term satisfies the inequality 16

$$
\left|\frac{f(a)+f(b)}{2}-\frac{1}{b-a} \int_{a}^{b} f(t) d t\right| \leq \frac{1}{4} L(b-a) .
$$

For related results see [1], [5]-[12, [14]-[17], [19]-23] and the references therein.

In the following, under the conditions of Theorem 1 , we use inequality (2.6) in the following form

$$
\|f(y)-f(x)\| \leq \frac{1}{2}\|y-x\|\left[f_{a}^{\prime}(\|x\|)+f_{a}^{\prime}(\|y\|)\right]
$$


for any $x, y \in \mathcal{B}$ with $\|x\|,\|y\|<R$, to derive some simple refinements of the Hermite-Hadamard type inequalities (4.1) and (4.2) for power series of elements in a unital Banach algebra.

TheOREM 3. Let $f(z)=\sum_{n=0}^{\infty} a_{n} z^{n}$ be a function defined by power series with complex coefficients and convergent on the open disk $D(0, R) \subset \mathbb{C}, R>0$. For any $x, y \in \mathcal{B}$ with $\|x\|,\|y\|<R$ we have

$$
\begin{aligned}
& \left\|f\left(\frac{x+y}{2}\right)-\int_{0}^{1} f((1-s) x+s y) d s\right\| \\
& \leq \frac{1}{2}\|y-x\|\left[\frac{1}{4} f_{a}^{\prime}\left(\left\|\frac{x+y}{2}\right\|\right)+\int_{0}^{1}\left|s-\frac{1}{2}\right| f_{a}^{\prime}(\|(1-s) x+s y\|) d s\right] \\
& \leq \frac{1}{8}\|y-x\|\left[f_{a}^{\prime}\left(\left\|\frac{x+y}{2}\right\|\right)+\frac{1}{2}\left[f_{a}^{\prime}(\|x\|)+f_{a}^{\prime}(\|y\|)\right]\right] \\
& \leq \frac{1}{8}\|y-x\|\left[f_{a}^{\prime}(\|x\|)+f_{a}^{\prime}(\|y\|)\right] \\
& \leq \frac{1}{4}\|y-x\| \max \left\{f_{a}^{\prime}(\|x\|), f_{a}^{\prime}(\|y\|)\right\}
\end{aligned}
$$

Proof. From the inequality 4.3 we have

$$
\begin{aligned}
\| f\left(\frac{x+y}{2}\right) & -f((1-s) x+s y) \| \\
& \leq \frac{1}{2}\left|s-\frac{1}{2}\right|\|y-x\|\left[f_{a}^{\prime}\left(\left\|\frac{x+y}{2}\right\|\right)+f_{a}^{\prime}(\|(1-s) x+s y\|)\right]
\end{aligned}
$$

for any $x, y \in \mathcal{B}$ with $\|x\|,\|y\|<R$ and $s \in[0,1]$. Integrating on $[0,1]$ we have

$$
\begin{aligned}
\left\|f\left(\frac{x+y}{2}\right)-\int_{0}^{1} f((1-s) x+s y) d s\right\| \\
\quad \leq \int_{0}^{1}\left\|f\left(\frac{x+y}{2}\right)-f((1-s) x+s y)\right\| d s \\
\quad \leq \frac{1}{2}\|y-x\|\left[\frac{1}{4} f_{a}^{\prime}\left(\left\|\frac{x+y}{2}\right\|\right)+\int_{0}^{1}\left|s-\frac{1}{2}\right| f_{a}^{\prime}(\|(1-s) x+s y\|) d s\right]
\end{aligned}
$$

and the first inequality in 4.4 is proved. 
Since the function $g(s):=f_{a}^{\prime}(\|(1-s) x+s y\|)$ is convex on $[0,1]$, then we have

$$
\begin{aligned}
& \int_{0}^{1}\left|s-\frac{1}{2}\right| f_{a}^{\prime}(\|(1-s) x+s y\|) d s \\
& \leq \int_{0}^{1}\left|s-\frac{1}{2}\right|\left[(1-s) f_{a}^{\prime}(\|x\|)+s f_{a}^{\prime}(\|y\|)\right] d s \\
& =f_{a}^{\prime}(\|x\|) \int_{0}^{1}\left|s-\frac{1}{2}\right|(1-s) d s+f_{a}^{\prime}(\|y\|) \int_{0}^{1}\left|s-\frac{1}{2}\right| s d s
\end{aligned}
$$

Since

$$
\int_{0}^{1}\left|s-\frac{1}{2}\right|(1-s) d s=\int_{0}^{1}\left|s-\frac{1}{2}\right| s d s=\frac{1}{8},
$$

then by 4.5 we have

$$
\int_{0}^{1}\left|s-\frac{1}{2}\right| f_{a}^{\prime}(\|(1-s) x+s y\|) d s \leq \frac{1}{8}\left[f_{a}^{\prime}(\|x\|)+f_{a}^{\prime}(\|y\|)\right]
$$

and the second inequality in (4.4) is proved.

The last part is obvious.

REMARK 4. If $x, y \in \mathcal{B}$ with $\|x\|,\|y\| \leq M<R$, then by the inequality (4.4) we have

$$
\left\|f\left(\frac{x+y}{2}\right)-\int_{0}^{1} f((1-s) x+s y) d s\right\| \leq \frac{1}{4} f_{a}^{\prime}(M)\|y-x\| .
$$

The trapezoidal version is as follows:

THEOREM 4. Let $f(z)=\sum_{n=0}^{\infty} a_{n} z^{n}$ be a function defined by power series with complex coefficients and convergent on the open disk $D(0, R) \subset \mathbb{C}, R>0$. For any $x, y \in \mathcal{B}$ with $\|x\|,\|y\|<R$ we have

$$
\begin{aligned}
& \left\|\frac{f(x)+f(y)}{2}-\int_{0}^{1} f((1-s) x+s y) d s\right\| \\
\leq & \frac{1}{8}\|y-x\|\left[\frac{f_{a}^{\prime}(\|x\|)+f_{a}^{\prime}(\|y\|)}{2}\right. \\
+ & \int_{0}^{1} s\left[f_{a}^{\prime}\left(\left\|(1-s) x+s \frac{x+y}{2}\right\|\right)+f_{a}^{\prime}\left(\left\|s \frac{x+y}{2}+(1-s) y\right\|\right)\right] d s
\end{aligned}
$$




$$
\begin{aligned}
& \leq \frac{1}{4}\|y-x\|\left[\frac{f_{a}^{\prime}(\|x\|)+f_{a}^{\prime}(\|y\|)}{3}+\frac{1}{3} f_{a}^{\prime}\left(\left\|\frac{x+y}{2}\right\|\right)\right] \\
& \leq \frac{1}{8}\|y-x\|\left[f_{a}^{\prime}(\|x\|)+f_{a}^{\prime}(\|y\|)\right] \leq \frac{1}{4}\|y-x\| \max \left\{f_{a}^{\prime}(\|x\|), f_{a}^{\prime}(\|y\|)\right\} .
\end{aligned}
$$

Proof. From the inequality 4.3 we have

$$
\begin{aligned}
& \left\|f(x)-f\left((1-s) x+s \frac{x+y}{2}\right)\right\| \\
& \quad \leq \frac{1}{4} s\|y-x\|\left[f_{a}^{\prime}(\|x\|)+f_{a}^{\prime}\left(\left\|(1-s) x+s \frac{x+y}{2}\right\|\right)\right]
\end{aligned}
$$

and

$$
\begin{aligned}
& \left\|f(y)-f\left(s \frac{x+y}{2}+(1-s) y\right)\right\| \\
& \quad \leq \frac{1}{4} s\|y-x\|\left[f_{a}^{\prime}(\|y\|)+f_{a}^{\prime}\left(\left\|s \frac{x+y}{2}+(1-s) y\right\|\right)\right]
\end{aligned}
$$

for any $x, y \in \mathcal{B}$ with $\|x\|,\|y\|<R$ and $s \in[0,1]$. Utilising the triangle inequality, 4.7) and 4.8 we have

$$
\begin{aligned}
\| \frac{f(x)+f(y)}{2}- & \frac{1}{2}\left[f\left((1-s) x+s \frac{x+y}{2}\right)+f\left(s \frac{x+y}{2}+(1-s) y\right)\right] \| \\
\leq & \frac{1}{2}\left\|f(x)-f\left((1-s) x+s \frac{x+y}{2}\right)\right\| \\
& +\frac{1}{2}\left\|f(y)-f\left(s \frac{x+y}{2}+(1-s) y\right)\right\| \\
\leq & \frac{1}{8} s\|y-x\|\left[f_{a}^{\prime}(\|x\|)+f_{a}^{\prime}\left(\left\|(1-s) x+s \frac{x+y}{2}\right\|\right)\right] \\
& +\frac{1}{8} s\|y-x\|\left[f_{a}^{\prime}(\|y\|)+f_{a}^{\prime}\left(\left\|s \frac{x+y}{2}+(1-s) y\right\|\right)\right]
\end{aligned}
$$

for any $x, y \in \mathcal{B}$ with $\|x\|,\|y\|<R$ and $s \in[0,1]$. 
Integrating on $[0,1]$ we have

$$
\text { (4.9) } \begin{aligned}
& \| \frac{f(x)+f(y)}{2} \\
&-\frac{1}{2} \int_{0}^{1}\left[f\left((1-s) x+s \frac{x+y}{2}\right)+f\left(s \frac{x+y}{2}+(1-s) y\right)\right] d s \| \\
& \leq \int_{0}^{1} \| \frac{f(x)+f(y)}{2} \\
&-\frac{1}{2}\left[f\left((1-s) x+s \frac{x+y}{2}\right)+f\left(s \frac{x+y}{2}+(1-s) y\right)\right] \| d s \\
& \leq \frac{1}{8}\|y-x\|\left[\frac{f_{a}^{\prime}(\|x\|)+f_{a}^{\prime}(\|y\|)}{2}\right. \\
&+\int_{0}^{1} s\left[f_{a}^{\prime}\left(\left\|(1-s) x+s \frac{x+y}{2}\right\|\right)+f_{a}^{\prime}\left(\left\|s \frac{x+y}{2}+(1-s) y\right\|\right)\right] d s=: J .
\end{aligned}
$$

By the convexity of the functions $h(s):=f_{a}^{\prime}\left(\left\|(1-s) x+s \frac{x+y}{2}\right\|\right)$ and $k(s):=$ $f_{a}^{\prime}\left(\left\|s \frac{x+y}{2}+(1-s) y\right\|\right)$ on the interval $[0,1]$ we have

$$
\begin{aligned}
\int_{0}^{1} s\left[f_{a}^{\prime}(\|(1-s) x+\right. & \left.\left.s \frac{x+y}{2} \|\right)+f_{a}^{\prime}\left(\left\|s \frac{x+y}{2}+(1-s) y\right\|\right)\right] d s \\
\leq & \int_{0}^{1} s\left[(1-s) f_{a}^{\prime}(\|x\|)+s f_{a}^{\prime}\left(\left\|\frac{x+y}{2}\right\|\right)\right] d s \\
& +\int_{0}^{1} s\left[s f_{a}^{\prime}\left(\left\|\frac{x+y}{2}\right\|\right)+(1-s) f_{a}^{\prime}(\|y\|)\right] d s \\
= & f_{a}^{\prime}(\|x\|) \int_{0}^{1} s(1-s) d s+2 f_{a}^{\prime}\left(\left\|\frac{x+y}{2}\right\|\right) \int_{0}^{1} s^{2} d s \\
& +f_{a}^{\prime}(\|y\|) \int_{0}^{1} s(1-s) d s \\
= & \frac{1}{6}\left[f_{a}^{\prime}(\|x\|)+f_{a}^{\prime}(\|y\|)\right]+\frac{2}{3} f_{a}^{\prime}\left(\left\|\frac{x+y}{2}\right\|\right) .
\end{aligned}
$$

Therefore

$$
J \leq \frac{1}{4}\|y-x\|\left[\frac{f_{a}^{\prime}(\|x\|)+f_{a}^{\prime}(\|y\|)}{3}+\frac{1}{3} f_{a}^{\prime}\left(\left\|\frac{x+y}{2}\right\|\right)\right] .
$$


Now, using the change of variable $t=2 s$ we have

$$
\frac{1}{2} \int_{0}^{1} f\left((1-t) x+t \frac{x+y}{2}\right) d t=\int_{0}^{1 / 2} f((1-s) x+s y) d s
$$

and by the change of variable $t=1-v$ we have

$$
\frac{1}{2} \int_{0}^{1} f\left(t \frac{x+y}{2}+(1-t) x\right) d t=\frac{1}{2} \int_{0}^{1} f\left((1-v) \frac{x+y}{2}+v y\right) d v .
$$

Moreover, if we make the change of variable $v=2 s-1$ we also have

$$
\frac{1}{2} \int_{0}^{1} f\left((1-v) \frac{x+y}{2}+v y\right) d v=\int_{1 / 2}^{1} f((1-s) x+s y) d s .
$$

Therefore

$$
\begin{aligned}
\frac{1}{2} \int_{0}^{1}[f((1-s) & \left.\left.x+s \frac{x+y}{2}\right)+f\left(s \frac{x+y}{2}+(1-s) y\right)\right] d s \\
& =\int_{0}^{1 / 2} f((1-s) x+s y) d t+\int_{1 / 2}^{1} f((1-s) x+s y) d s \\
& =\int_{0}^{1} f((1-s) x+s y) d t .
\end{aligned}
$$

Utilising (4.9) and (4.10) we deduce the first two inequalities in 4.6). The rest is obvious.

REMARK 5. If $x, y \in \mathcal{B}$ with $\|x\|,\|y\| \leq M<R$, then by the inequality 4.6. we have

$$
\left\|\frac{f(x)+f(y)}{2}-\int_{0}^{1} f((1-s) x+s y) d s\right\| \leq \frac{1}{4} f_{a}^{\prime}(M)\|y-x\| .
$$

REMARK 6. Some vector norm inequalities for power series of operators in Hilbert spaces that are similar to the results from Theorems 3 and 4 above may be found in [3, Corollary 3]. 
For any $x, y \in \mathcal{B}$ we have the following sequence of midpoint inequalities for the exponential function

$$
\begin{aligned}
& \text { 11) }\left\|\exp \left(\frac{x+y}{2}\right)-\int_{0}^{1} \exp ((1-s) x+s y) d s\right\| \\
& \leq \frac{1}{2}\|y-x\|\left[\frac{1}{4} \exp \left(\left\|\frac{x+y}{2}\right\|\right)+\int_{0}^{1}\left|s-\frac{1}{2}\right| \exp (\|(1-s) x+s y\|) d s\right] \\
& \leq \frac{1}{8}\|y-x\|\left[\exp \left(\left\|\frac{x+y}{2}\right\|\right)+\frac{1}{2}[\exp (\|x\|)+\exp (\|y\|)]\right] \\
& \leq \frac{1}{8}\|y-x\|[\exp (\|x\|)+\exp (\|y\|)] \\
& \leq \frac{1}{4}\|y-x\| \max \{\exp (\|x\|), \exp (\|y\|)\} .
\end{aligned}
$$

We also have the trapezoid type inequalities

$$
\begin{aligned}
& \text { (4.12) }\left\|\frac{\exp (x)+\exp (y)}{2}-\int_{0}^{1} \exp ((1-s) x+s y) d s\right\| \\
& \leq \frac{1}{8}\|y-x\|\left[\frac{\exp (\|x\|)+\exp (\|y\|)}{2}\right.
\end{aligned}
$$$$
\left.+\int_{0}^{1} s\left[\exp \left(\left\|(1-s) x+s \frac{x+y}{2}\right\|\right)+\exp \left(\left\|s \frac{x+y}{2}+(1-s) y\right\|\right)\right] d s\right]
$$$$
\leq \frac{1}{4}\|y-x\|\left[\frac{\exp (\|x\|)+\exp (\|y\|)}{3}+\frac{1}{3} \exp \left(\left\|\frac{x+y}{2}\right\|\right)\right]
$$

$\leq \frac{1}{8}\|y-x\|[\exp (\|x\|)+\exp (\|y\|)]$

$\leq \frac{1}{4}\|y-x\| \max \{\exp (\|x\|), \exp (\|y\|)\}$.

It is known that if $x$ and $y$ are commuting, i.e. $x y=y x$, then the exponential function satisfies the property

$$
\exp (x) \exp (y)=\exp (y) \exp (x)=\exp (x+y) .
$$

Also, if $x$ is invertible and $a, b \in \mathbb{R}$ with $a<b$ then

$$
\int_{a}^{b} \exp (t x) d t=x^{-1}[\exp (b x)-\exp (a x)] .
$$


Moreover, if $x$ and $y$ are commuting and $y-x$ is invertible, then

$$
\begin{aligned}
\int_{0}^{1} \exp ((1-s) x+s y) d s & =\int_{0}^{1} \exp (s(y-x)) \exp (x) d s \\
& =\left(\int_{0}^{1} \exp (s(y-x)) d s\right) \exp (x) \\
& =(y-x)^{-1}[\exp (y-x)-I] \exp (x) \\
& =(y-x)^{-1}[\exp (y)-\exp (x)] .
\end{aligned}
$$

In this case the first term in 4.11 may be replaced by

$$
\left\|\exp \left(\frac{x+y}{2}\right)-(y-x)^{-1}[\exp (y)-\exp (x)]\right\|
$$

while the first term in 4.12 , by

$$
\left\|\frac{\exp (x)+\exp (y)}{2}-(y-x)^{-1}[\exp (y)-\exp (x)]\right\| .
$$

The interested reader may apply the above inequalities to other important functions such as $(1-z)^{-1}=\sum_{n=0}^{\infty} z^{n}$ and $(1+z)^{-1}=\sum_{n=0}^{\infty}(-1)^{n} z^{n}$ defined on $D(0,1)$. However, the details are omitted.

Acknowledgement. The author would like to thank the anonymous referee for valuable suggestions that have been implemented in the final version of this paper.

\section{References}

[1] Azpeitia A.G., Convex functions and the Hadamard inequality, Rev. Colombiana Mat. 28 (1994), no. 1, 7-12.

[2] Bhatia R., Matrix analysis, Springer-Verlag, New York, 1997.

[3] Cheung W.-S., Dragomir S.S., Vector norm inequalities for power series of operators in Hilbert spaces, Tbilisi Math. J. 7 (2014), no. 2, 21-34.

[4] Dragomir S.S., Cho Y.J., Kim S.S., Inequalities of Hadamard's type for Lipschitzian mappings and their applications, J. Math. Anal. Appl. 245 (2000), no. 2, 489-501.

[5] Dragomir S.S., A mapping in connection to Hadamard's inequalities, Anz. Österreich. Akad. Wiss. Math.-Natur. Kl. 128 (1991), 17-20.

[6] Dragomir S.S., Two mappings in connection to Hadamard's inequalities, J. Math. Anal. Appl. 167 (1992), 49-56. 
[7] Dragomir S.S., On Hadamard's inequalities for convex functions, Math. Balkanica 6 (1992), 215-222.

[8] Dragomir S.S., An inequality improving the second Hermite-Hadamard inequality for convex functions defined on linear spaces and applications for semi-inner products, J. Inequal. Pure Appl. Math. 3 (2002), no. 3, Art. 35.

[9] Dragomir S.S., Bounds for the normalized Jensen functional, Bull. Austral. Math. Soc. 74 (2006), 471-476.

[10] Dragomir S.S., Gomm I., Bounds for two mappings associated to the HermiteHadamard inequality, Aust. J. Math. Anal. Appl. 8 (2011), Art. 5, 9 pp.

[11] Dragomir S.S., Gomm I., Some new bounds for two mappings related to the HermiteHadamard inequality for convex functions, Numer. Algebra Cont Optim. 2 (2012), no. 2, 271-278.

[12] Dragomir S.S., Milośević D.S., Sándor J., On some refinements of Hadamard's inequalities and applications, Univ. Belgrad, Publ. Elek. Fak. Sci. Math. 4 (1993), 21-24.

[13] Dragomir S.S., Pearce C.E.M., Selected topics on Hermite-Hadamard inequalities and applications, RGMIA Monographs, 2000. Available at http://rgmia.org/monographs/ hermite_hadamard.html

[14] Guessab A., Schmeisser G., Sharp integral inequalities of the Hermite-Hadamard type, J. Approx. Theory 115 (2002), no. 2, 260-288.

[15] Kilianty E., Dragomir S.S., Hermite-Hadamard's inequality and the p-HH-norm on the Cartesian product of two copies of a normed space, Math. Inequal. Appl. 13 (2010), no. 1, 1-32.

[16] Matić M., Pečarić J., Note on inequalities of Hadamard's type for Lipschitzian mappings, Tamkang J. Math. 32 (2001), no. 2, 127-130.

[17] Merkle M., Remarks on Ostrowski's and Hadamard's inequality, Univ. Beograd. Publ. Elektrotehn. Fak. Ser. Mat. 10 (1999), 113-117.

[18] Mikusiński J., The Bochner integral, Birkhäuser Verlag, Basel, 1978.

[19] Pearce C.E.M., Rubinov A.M., P-functions, quasi-convex functions, and Hadamard type inequalities, J. Math. Anal. Appl. 240 (1999), no. 1, 92-104.

[20] Pečarić J., Vukelić A., Hadamard and Dragomir-Agarwal inequalities, the Euler formulae and convex functions, in: Functional equations, inequalities and applications, Kluwer Acad. Publ., Dordrecht, 2003, pp. 105-137.

[21] Toader G., Superadditivity and Hermite-Hadamard's inequalities, Studia Univ. BabeşBolyai Math. 39 (1994), no. 2, 27-32.

[22] Yang G.-S., Hong M.-C., A note on Hadamard's inequality, Tamkang J. Math. 28 (1997), no. 1, 33-37.

[23] Yang G.-S., Tseng K.-L., On certain integral inequalities related to Hermite-Hadamard inequalities, J. Math. Anal. Appl. 239 (1999), no. 1, 180-187.

Mathematics, School of Engineering \& Science

Victoria University

PO Box 14428

Melbourne City, MC 8001

Australia

AND

School of Computer Science \& Applied Mathematics

University OF THE WitwatersRand

Private Bag 3

JoHANNESBURG 2050

South Africa

e-mail: sever.dragomir@vu.edu.au

URL: http://rgmia.org/dragomir 